\title{
Dynamics of Agglomeration of Magnetite Nanoparticles under Magnetic Field Studied by Monitoring Magnetic Weight
}

\author{
Daeseong Jin and Hackjin Kim* \\ Department of Chemistry, Chungnam National University \\ Taejeon 305-764, Korea \\ hackjin@cnu.ac.kr
}

\section{Extended Abstract}

One of many important applications of magnetic nanoparticles is the separation of complex mixture by magnetic field [1]. Magnetic nanoparticles aggregate with impurities of the mixture under magnetic field in the separation process. We have built a simple experimental setup for monitoring the temporal change of magnetic weight with a conventional electronic balance in order to study the agglomeration dynamics of magnetite nanoparticles by magnetic field [2]. The magnetic field is applied in the direction parallel to the gravitational force in our setup. Magnetite nanoparticles are prepared by reacting $\mathrm{FeCl}_{2}$ and $\mathrm{FeCl}_{3}$ in aqueous ammonia solution [3].

When the ferrofluid sample is placed under the field, the magnetic weight of the sample jumps instantaneously by Neel and Brown relaxation, and then increases slowly over a few days as the nanoparticles agglomerate. The slow increase of the magnetic weight shows the stretched exponential behaviour, $M(t)=M(\infty)+[M(0)-M(\infty)] \exp \left[-(t / \tau)^{\beta}\right]$ where $0<\beta$ $<1$. The stretched exponential function results from the distribution of energy barrier involved in the dynamics, which can be calculated by the inverse Laplace transformation of the stretched exponential function if the Arrhenius pre-exponent factor of the rate constant is given [4]. Typical agglomeration of magnetite nanoparticles by magnetic field gives the energy barrier distribution function with the peak energy of $\sim 36 \mathrm{~kJ} \mathrm{~mol}^{-1}$ and the width of $\sim 8 \mathrm{~kJ} \mathrm{~mol}^{-1}$ [5]. In addition to the overall stretched exponential change, the magnetic weight of ferrofluid shows thermal fluctuation, which is explained well with the Boltzmann factor. The energy difference of $<10 \mathrm{~kJ} \mathrm{~mol}^{-1}$, estimated from the temperature dependence of the dynamics, that is, the Boltzmann factor corresponds to the energy difference between the agglomerated states. Again, this energy difference is not a single value but shows some distribution.

\section{References}

[1] C. T. Yavuz, J. T. Mayo, W. W. Yu, A. Prakash, J. C. Falkner, S. Yean, L. Cong, H. J. Shipley, A. Kan, M. Tomson, D. Natelson, and V. L. Colvin, "Low-Field Magnetic Separation of Monodisperse $\mathrm{Fe}_{3} \mathrm{O}_{4}$ Nanocrystals," Science, vol. 314, pp. 964-967, 2006.

[2] D. Jin and H. Kim, "Magnetization of Magnetite Ferrofluid Studied by Using a Magnetic Balance," Bull. Korean Chem. Soc., vol. 34, no. 6, pp. 1715-1721, 2013.

[3] Y. S. Kang, S. Risbud, J. F. Rabolt, and P. Stroeve, "Synthesis and Characterization of Nanometer-Size $\mathrm{Fe}_{3} \mathrm{O}_{4}$ and $\gamma$ $\mathrm{Fe}_{2} \mathrm{O}_{3}$ Particles," Chem. Mater., vol. 8, pp. 2209-2211, 1996.

[4] R. H. Austin, K. Beeson, L. Eisenstein, H. Frauenfelder, I. C. Gunsalus, and V. P. Marshall, "Activation Energy Spectrum of a Biomolecule: Photodissociation of Carbonmonoxy Myoglobin at Low Temperatures," Phys. Rev. Lett., vol. 32, no. 8, pp. 403-405, 1974.

[5] D. Jin and H. Kim, "Stretched Exponential Change of Magnetic Weight of Magnetite Ferrofluid: Distribution of Energy Barrier for Agglomeration of Nanoparticles," Bull. Korean Chem. Soc., vol. 36, pp. 424-426, 2015. 